\title{
Tretinoin Enhances Minoxidil Response in Androgenetic Alopecia Patients by Up-Regulating Follicular Sulfotransferase Enzymes
}

\author{
Aseem Sharma, Andy Goren, Rachita Dhurat, Sandip Agrawal, Rodney \\ Sinclair, Ralph Trueb, Sergio Vano-Galvan, Guangping Chen, Yimei \\ Tan, Maja Kovacevic, Mirna Situm, John McCoy*
}

*Author whom correspondence should be addressed (johnm@appliedbiology.com)

\section{Author Affiliations:}

Aseem Sharma, MD: Department of Dermatology, LTM Medical College \& Hospital Sion, Mumbai, India.

Andy Goren, MD: Applied Biology, California, USA. Department of Dermatology and Venereology, University Hospital Center “Sestre milosrdnice”, Zagreb, Croatia.

Rachita Dhurat, MD: Department of Dermatology, LTM Medical College \& Hospital Sion, Mumbai, India.

Sandip Agrawal, MD: Department of Dermatology, LTM Medical College \& Hospital Sion, Mumbai, India.

Rodney Sinclair, MD: Department of Medicine, University of Melbourne, Victoria, Australia.

Ralph M. Trüeb, MD: Center for Dermatology and Hair Diseases, Zurich-Wallisellen, Switzerland.

Sergio Vañó-Galván, MD, PhD: Trichology Unit. Dermatology Department. Ramon y Cajal Hospital, Madrid, Spain.

Guangping Chen, PhD: Department of Physiological Sciences, Oklahoma State University, Oklahoma, USA.

Yimei Tan, MD: Skin \& Cosmetic Research Department, Shanghai Skin Disease Hospital, Shanghai, China.

This is the author manuscript accepted for publication and has undergone full peer review but has not been through the copyediting, typesetting, pagination and proofreading process, which may lead to differences between this version and the Version of Record. Please cite this article as doi: $10.1111 /$ dth.12915

This article is protected by copyright. All rights reserved. 
Maja Kovacevic, MD: Department of Dermatology and Venereology, University Hospital Center "Sestre milosrdnice", Zagreb, Croatia.

Mirna Situm, MD: Department of Dermatology and Venereology, University Hospital Center "Sestre milosrdnice”, Zagreb, Croatia.

John McCoy, PhD: Applied Biology, California, USA.

\begin{abstract}
Minoxidil sulfate is the active metabolite required to exert the vasodilatory and hair growing effects of minoxidil. For hair growth, sulfotransferase enzymes expressed in outer root sheath of the hair follicle sulfonate minoxidil. The large intra-subject variability in follicular sulfotransferase was found to predict minoxidil response and thus explain the low response rate to topical minoxidil in the treatment of androgenetic alopecia. A method to increase minoxidil response would be of significant clinical utility. Retinoids have been reported to increase minoxidil response. The purported mechanism of action was retinoid modulation of skin permeation to minoxidil, however, evidence to the contrary supports retinoids increase dermal thickness. In order to elucidate the effect of topical retinoids on minoxidil response, we studied the effect of topical tretinoin on follicular sulfotransferase. In this study, we demonstrate that topical tretinoin application influences the expression of follicular sulfotransferase. Of clinical significance, in our cohort, $43 \%$ of subjects initially predicted to be non-responders to minoxidil were converted to responders following 5 days of topical tretinoin application. To our knowledge, this is the first study to elucidate the interaction mechanism between topical minoxidil and retinoids and thus provides a pathway for the development of future androgenetic alopecia treatments.
\end{abstract}

\title{
BACKGROUND
}

In the late 1970s, an oral hypotensive agent, minoxidil, was undergoing clinical studies in support of US FDA approval. Hypertrichosis was a common adverse event frequently experienced by study participants. Subsequently, the UpJohn corporation launched a drug development program that culminated in the approval of the first topical drug treatment for androgenetic alopecia, $2 \%$ topical minoxidil solution (1). While the clinical impact on some androgenetic alopecia patients has been enormous, less than $40 \%$ of the patients observe a significant clinical response to topical minoxidil (2). 
Minoxidil sulfate is the active metabolite responsible for the vasodilatory and hair growth promoting effects of minoxidil (3). Sulfotransferase enzymes in the outer root sheath of the hair follicle convert minoxidil to its active form. In a number of studies sampling over 200 subjects, our group has demonstrating the clinical utility and validity of a test to measure the follicular sulfotransferase enzymatic activity in plucked hair as a method to predict minoxidil response (46). The test demonstrates an accuracy of $97.8 \%$ in identifying non-responders to minoxidil (4). A logical progression of this work would be to find agents that can increase the activity of follicular sulfotransferases and hence increase minoxidil response. Accordingly, we have been in pursuit of an adjuvant therapy to clinically improve minoxidil response through modulation of the sulfotransferase enzymatic activity.

Topical tretinoin (all-trans-retinoic acid) has been suggested as a promoter of hair growth to be used alone or in combination with minoxidil (7). In-vitro studies demonstrate a synergistic increase in the expression of hair growth markers (e.g., Erk, Akt, Bcl-2 and Bax) after treatment with a combination of tretinoin and minoxidil (8-9). Additionally, clinical evidence suggests that enhanced systematic absorption of minoxidil was detected in subject using a combination of tretinoin and minoxidil (10). A mechanism of reduced stratum corneum barrier function was suggested as the reason for increased minoxidil absorption, however, evidence to the contrary indicates tretinoin increases dermal thickness (11). This discrepancy led us to theorize that the increased absorption of minoxidil may be the result of increased sulfonation. In-vitro studies support this, as all-trans retinoic acid has been shown to induce sulfotransferase in human hepatic carcinoma cells (12).

\section{METHODS}

Twenty subjects 18 years of age or older (10 males and 10 females) were recruited at a hospital dermatology department. The study was conducted under review of the hospital ethics committee. All subjects gave informed consent prior to joining the study. The average age of all subjects was 32. The average Norwood score for the male subjects was III and the average Ludwig score for the female subjects was II. All subjects were diagnosed with androgenetic alopecia. Subjects presenting with seborrheic dermatitis, scalp psoriasis, or other scalp disorders were excluded from the study. None of the subjects had used minoxidil, finasteride, or a retinoid (topical or oral) during the past 6 months.

A target area $(10 \mathrm{~cm} \mathrm{x} 10 \mathrm{~cm})$ on the scalp of each subject was identified. The target area was marked with a surgical pen. At baseline, 10 hairs with visible bulbs were plucked from the target area. The hairs were analyzed using the Minoxidil Response Test (Follea Ltd., Munich, Germany), an in-vitro medical device for determining a patient's response to topical minoxidil. The test procedure has been described previously (4). The value from the test indicates the level of sulfotransferase activity present in the sampled hairs, a value less than 0.4 indicates a person does not have adequate sulfotransferase activity to respond to topical minoxidil. Each data point 
is an average of values from 4 samples. Subsequently, the principal investigator applied approximately $2 \mathrm{mg} / \mathrm{cm}^{2}$ of a topical cream containing $0.1 \%$ tretinoin to the target area.

Each subject was supplied with a $20 \mathrm{~g}$ tube of topical tretinoin $0.1 \%$ and was instructed to apply the topical cream to the target area once a day for four consecutive days. The subjects were instructed to ensure their scalp was dry prior to the tretinoin application. A total of 5 applications (including the baseline application) were applied to each subject's scalp. On day 6, each subject returned to the clinic and the principal investigator collected 10 plucked hairs with visible bulbs from the target area, as well as, inspected the target area for any adverse reaction. Hairs collected at the final visit were tested with the Minoxidil Response Test for comparison.

\section{RESULTS}

All twenty subjects completed the study. No adverse events were reported by the subjects, nor did the principal investigator observe any adverse reactions at the conclusion of the study. Data from the Minoxidil Response Test before and after 5 days of treatment with the tretinoin cream are shown in Table 1. The data are tabulated according to the MRT value measured at baseline. For the entire cohort, prior to treatment, the mean sulfotransferase enzymatic activity as measured by the Minoxidil Response Test was $0.57 \pm 0.33$ (mean \pm s.d.). Following 5 days of treatment with topical $0.1 \%$ tretinoin, the mean sulfotransferase enzymatic activity was $0.58 \pm 0.29$. The one-sided paired t-test yielded a $\mathrm{p}=0.187$ (MedCalc v18.11.3); thus, for the entire cohort, topical tretinoin does not appear to significantly induce the follicular sulfotransferase enzymes. However, a further analysis of the data based on subjects predicted to be non-responders to topical minoxidil, i.e., those with a test value less than 0.4 , yielded a pretreatment enzymatic activity of $0.26 \pm 0.09$ and a post-treatment value of $0.35 \pm 0.16$. The onesided paired t-test achieved statistical significance with $\mathrm{p}=0.0397$ (MedCalc v18.11.3). Thus, among non-responders in our cohort, five days of treatment with topical $0.1 \%$ tretinoin significantly increased follicular sulfotransferase enzymatic activity. More importantly, following five days of treatment with topical $0.1 \%$ tretinoin, $43 \%$ of the subjects originally predicted to be non-responders to topical minoxidil were converted to responders.

\section{DISCUSSION}

Topical minoxidil is the only US FDA approved topical drug for the treatment of androgenetic alopecia. Unfortunately, the clinical response to topical minoxidil remains low, $30 \%-40 \%$ (2). Several reports have suggested that combining topical minoxidil with a topical tretinoin may increase hair growth (8-10). Additionally, demonstrations of enhanced minoxidil absorption after treatment with tretinoin led to the suggestion that tretinoin lowered the barrier function of the dermis (10). However, this explanation is unlikely, as retinoids tend to increase dermal thickness (11). Here we report that tretinoin treatment increases the sulfonation activity of the hair follicle. Interestingly, increased follicular sulfonation would likely produce an 
increase in detectable minoxidil in the blood, as minoxidil sulfate is more water-soluble than minoxidil. This would explain previous reports, however, would not support the conclusion that tretinoin decrease barrier function of the skin.

In our previous work, we have demonstrated that the enzymatic activity of the sulfotransferase enzymes in hair follicles predicts minoxidil response (4-6). In this study, we further built on our findings and demonstrated that topical tretinoin application up-regulates follicular sulfotransferase enzymes for subjects with low basal expression. Of clinical significance, $43 \%$ of subjects initially predicted to be non-responders to topical minoxidil were converted to responders following 5 days of topical tretinoin application. Sulfotransferase has been reported to be regulated by several nuclear factors including retinoic acid receptor (RXR), pregnane $\mathrm{X}$ receptor (PXR), and the constitutive androstane receptor (CAR), as well as, many others (12-13). All-trans retinoic acid is a well known agonist of the RXR nuclear receptor. RXR has also forms dimers with PXR and CAR to enhance induction of sulfotransferase (13). This work suggests that activation of the RXR nuclear receptor can up-regulate sulfotransferase in-vivo. Our group is currently exploring other nuclear receptor agonists and any synergies that may exist. This study illuminates a clear path to the development of an adjuvant therapy to increase the efficacy of topical minoxidil for the treatment of androgenetic alopecia.

\section{REFERENCES}

1. US FDA NDA 019501 Medical Review.

2. Olsen, E.A., Whiting, D., Bergfeld, W., Miller, J., Hordinsky, M., Wanser, R., Zhang, P., Kohut, B. (2007). A multicenter, randomized, placebo-controlled, double-blind clinical trial of a novel formulation of $5 \%$ minoxidil topical foam versus placebo in the treatment of androgenetic alopecia in men. Journal of the American Academy of Dermatology, 57, 767-774.

3. Buhl, A.E.,Waldon, D.J., Baker, C.A., Johnson, G.A. (1990). Minoxidil sulfate is the active metabolite that stimulates hair follicles. Journal of Investigative Dermatology, 95, 553-577.

4. Goren, A., Shapiro, J., Roberts, J., McCoy, J., Desai, N., Zarrab, Z., Pietrzak, A., Lotti, T. (2015). Clinical utility and validity of minoxidil response testing in androgenetic alopecia. Dermatologic Therapy, 28, 13-16.

5. Chitalia, J., Dhurat, R., Goren, A., McCoy, J., Kovacevic, M., Situm, M., Naccarato, T., Lotti, T. (2018). Characterization of follicular minoxidil sulfotransferase activity in a cohort of pattern hair loss patients from the Indian Subcontinent. Dermatologic Therapy, 31, e12741.

6. Goren, A., McCoy, J., Kovacevic, M., Situm, M., Chitalia, J., Dhurat, R., Naccarato, T., Lotti, T. (2018). The effect of topical minoxidil treatment on follicular sulfotransferase enzymatic activity. Journal of Biological Regulators and Homeostatic Agents, 32, 937-940.

7. Bazzano, G.,S., Terezakis, N., Galen, W. (1986). Topical tretinoin for hair growth promotion. Journal of American Academy of Dermatology, 15, 880-883. 
8. Kwon, O., S., Pyo, H., K., Oh, Y., J., Han, J., H., Lee, S., R., Chung, J., H., Eun, H., C., Kim, K., H. (2007). Promotive effect of minoxidil combined with all-trans retinoic acid (tretinoin) on human hair growth in vitro. Journal of Korean Medical Science, 22, 283-289.

9. Yoo, H., G., Chang, I., Y., Pyo, H., K., Kang, Y., J., Lee, S., H., Kwon, O., S., Cho, K., H., Eun, H., C., Kim, K., H. (2007). The additive effects of minoxidil and retinol on human hair growth in vitro. Biological and Pharmaceutical Bulletin, 30, 21-26.

10. Ferry, J., J., Forbes, K., K., VanderLugt, J., T., Szpunar, G., J. (1990). Influence of tretinoin on the percutaneous absorption of minoxidil from an aqueous topical solution. Clinical Pharmacology \& Therapeutics, 47, 439-446.

11. Voorhees, J., J. (1990). Clinical effects of long-term therapy with topical tretinoin and cellular mode of action. Journal of International Medical Research, 18, 26-28.

12. Maiti, S., Chen, X., Chen, G. (2005). All-trans retinoic acid induction of sulfotransferases. Basic \& Clinical Pharmacology \& Toxicology, 96, 44-53.

13. Kodama, S., Negishi, M. (2013). Sulfotransferase genes: regulation by nuclear receptors in response to xeno/endo-biotics. Drug Metabolism Reviews, 45, 441-449. 


\begin{tabular}{|c|c|c|c|c|}
\cline { 2 - 5 } \multicolumn{1}{c|}{} & \multicolumn{2}{|c|}{ Baseline } & \multicolumn{2}{c|}{5 Day Tretinoin } \\
\hline Subject ID & MRT & Response & MRT & Response \\
\hline 1 & 0.082 & NR & 0.079 & NR \\
2 & 0.221 & NR & 0.282 & NR \\
3 & 0.250 & NR & 0.425 & $\mathrm{R}$ \\
4 & 0.290 & NR & 0.575 & $\mathrm{R}$ \\
5 & 0.310 & NR & 0.277 & NR \\
6 & 0.324 & NR & 0.412 & $R$ \\
7 & 0.343 & NR & 0.383 & NR \\
8 & 0.419 & $R$ & 1.042 & $R$ \\
9 & 0.433 & $R$ & 0.405 & $R$ \\
10 & 0.439 & $R$ & 0.487 & $R$ \\
11 & 0.495 & $R$ & 0.615 & $R$ \\
12 & 0.558 & $R$ & 0.538 & $R$ \\
13 & 0.605 & $R$ & 1.077 & $R$ \\
14 & 0.678 & $R$ & 0.459 & $R$ \\
15 & 0.766 & $R$ & 0.365 & NR \\
16 & 0.806 & $R$ & 0.743 & $R$ \\
17 & 0.968 & $R$ & 0.493 & $R$ \\
18 & 1.033 & $R$ & 1.008 & $R$ \\
19 & 1.158 & $R$ & 0.861 & $R$ \\
20 & 1.203 & $R$ & 1.082 & $R$ \\
\hline
\end{tabular}

Table 1. The value of the Minoxidil Response Test (MRT) and the predicted minoxidil response of each subject $(\mathrm{R}=$ responder, $\mathrm{NR}=$ non-responder) before and after 5-day application of $0.1 \%$ tretinoin.

This article is protected by copyright. All rights reserved. 


\begin{tabular}{|c|c|c|c|c|}
\cline { 2 - 5 } \multicolumn{1}{c|}{} & \multicolumn{2}{c|}{ Baseline } & \multicolumn{2}{c|}{ 5 Day Tretinoin } \\
\hline Subject ID & MRT & Response & MRT & Response \\
\hline 1 & 0.082 & NR & 0.079 & NR \\
2 & 0.221 & NR & 0.282 & NR \\
3 & 0.250 & NR & 0.425 & $\mathrm{R}$ \\
4 & 0.290 & NR & 0.575 & $\mathrm{R}$ \\
5 & 0.310 & NR & 0.277 & NR \\
6 & 0.324 & NR & 0.412 & $\mathrm{R}$ \\
7 & 0.343 & NR & 0.383 & NR \\
8 & 0.419 & $\mathrm{R}$ & 1.042 & $\mathrm{R}$ \\
9 & 0.433 & $\mathrm{R}$ & 0.405 & $\mathrm{R}$ \\
10 & 0.439 & $\mathrm{R}$ & 0.487 & $\mathrm{R}$ \\
11 & 0.495 & $\mathrm{R}$ & 0.615 & $\mathrm{R}$ \\
12 & 0.558 & $\mathrm{R}$ & 0.538 & $\mathrm{R}$ \\
13 & 0.605 & $\mathrm{R}$ & 1.077 & $\mathrm{R}$ \\
14 & 0.678 & $\mathrm{R}$ & 0.459 & $\mathrm{R}$ \\
15 & 0.766 & $\mathrm{R}$ & 0.365 & $\mathrm{NR}$ \\
16 & 0.806 & $\mathrm{R}$ & 0.743 & $\mathrm{R}$ \\
17 & 0.968 & $\mathrm{R}$ & 0.493 & $\mathrm{R}$ \\
18 & 1.033 & $\mathrm{R}$ & 1.008 & $\mathrm{R}$ \\
19 & 1.158 & $\mathrm{R}$ & 0.861 & $\mathrm{R}$ \\
20 & 1.203 & $\mathrm{R}$ & 1.082 & $\mathrm{R}$ \\
\hline
\end{tabular}

Table 1. The value of the Minoxidil Response Test (MRT) and the predicted minoxidil response of each subject $(\mathrm{R}=$ responder, $\mathrm{NR}=$ non-responder $)$ before and after 5 -day application of $0.1 \%$ tretinoin.

This article is protected by copyright. All rights reserved. 


\section{University Library}

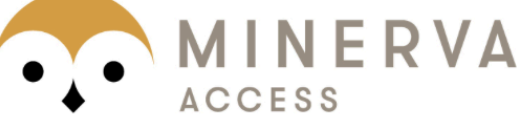

A gateway to Melbourne's research publications

Minerva Access is the Institutional Repository of The University of Melbourne

\section{Author/s:}

Sharma, A;Goren, A;Dhurat, R;Agrawal, S;Sinclair, R;Trueeb, RM;Vano-Galvan, S;Chen, G;Tan, Y;Kovacevic, M;Situm, M;McCoy, J

Title:

Tretinoin enhances minoxidil response in androgenetic alopecia patients by upregulating follicular sulfotransferase enzymes

Date:

2019-05-01

Citation:

Sharma, A., Goren, A., Dhurat, R., Agrawal, S., Sinclair, R., Trueeb, R. M., Vano-Galvan, S., Chen, G., Tan, Y., Kovacevic, M., Situm, M. \& McCoy, J. (2019). Tretinoin enhances minoxidil response in androgenetic alopecia patients by upregulating follicular sulfotransferase enzymes. DERMATOLOGIC THERAPY, 32 (3), https://doi.org/10.1111/dth.12915.

Persistent Link:

http://hdl.handle.net/11343/285774 